\title{
Aproximación al estudio evolutivo de las formas organizacionales
}

\section{Approach to the evolutionary study of organizational forms}

\author{
Henríquez Barráez, Aracely \\ Universidad Nacional Experimental Francisco de Miranda, Venezuela \\ Narváez Castro, Mercy \\ Universidad del Zulia, Venezuela
}

Autor por correspondencia: henriquezara@gmail.com, mercynarvaez@gmail.com

Fecha de recepción: 1 de Julio de 2018 - Fecha de aceptación: 10 de Septiembre de 2018

Resumen: El objetivo de este artículo es presentar por medio de un análisis documental una descripción de la evolución de las principales formas organizacionales adoptadas por las empresas, a partir de la contrastación teórica de los aportes realizados por autores como Chiavenato (2002), Rivas (2002), Padilla y Del Aguila (2003), Velarde, et al. (2003), así también como valor agregado se proponen algunos elementos constitutivos de una forma organizacional alternativa sistémica resultante de la reflexión de los de las configuraciones estructurales presentadas. Entre las formas organizacionales descritas se encuentran: el modelo de orgánico de Mintzberg; la organización horizontal, organización tipo red, organización celular y la organización virtual. Producto de la valoración de las necesidades organizacionales en los tiempos contemporáneos se concluye que las organizaciones deben abandonar las formas rígidas, burocráticas y normativas para adoptar una configuración más adaptativa, con pensamiento estratégico, manejo de la autoridad y poder descentralizado, con bases en el consenso, la cooperación y cuya característica principal sea la flexibilidad.

Palabras claves: formas organizacionales; elementos constitutivos; cooperación; flexibilidad

\begin{abstract}
The objective of this article is to present through a documentary analysis a description of the main organizational forms adopted by companies, based on the theoretical comparison of the contributions made by authors such as Chiavenato (2002), Rivas (2002), Padilla and Del Aguila (2003), Velarde, et al. (2003), as well as added value propose some constitutive elements of an alternative systemic organizational form resulting from the reflection of the presented structural configurations. Among the organizational forms described are: the Mintzberg's organic model; horizontal organization, network type organization, cellular organization and virtual organization. Product of the assessment of organizational needs in contemporary times, it is concluded that organizations must abandon the rigid, bureaucratic and normative forms to adopt a more adaptive configuration, with strategic thinking, management of authority and decentralized power, based on consensus, cooperation and whose main characteristic must be flexibility.
\end{abstract}

Key Words: organizational forms; constitutive elements; cooperation; flexibility 


\section{Introducción}

Para entender las demandas organizacionales del mundo globalizado, cambiante y competitivo, así como el tipo de organización que se desea configurar, es necesario comprender el pasado, el transitar que han tenido los sectores empresariales, así como también la evolución de lo que se constituyó como las bases del desarrollo organizacional y la teoría administrativa; aunado a una evaluación objetiva de la situación actual, del entorno y los factores influyentes en las organizaciones.

Tomando en consideración los aspectos teóricos que fundamentan la teoría administrativa en el campo gerencial, surge la inquietud de realizar esta investigación documental que tiene como fin describir la evolución de las principales formas organizacionales adoptadas por las empresas, esto se llevará a cabo realizando una contrastación de la perspectiva de cinco autores. El artículo se encuentra estructurado en dos secciones, la primera denominada "Configuraciones Estructurales de las Formas Organizativas", subdividida en 5 apartados, seguida de una segunda sección, llamada Propuesta Reflexiva.

\section{Métodos}

El proceso investigativo para el desarrollo de este artículo se abordó con una investigación documental en la que por medio de la descripción, explicación y contrastación de las teorías planteadas por los autores citados, se generó una propuesta reflexiva con representación en los elementos dinamizadores y acopladores de la forma organizacional. Entre los métodos de razonamiento lógico utilizados en la investigación se encuentran la deducción, empleada en el análisis de contenido y la abducción la cual permitió la construcción de la propuesta de los elementos del concepto organizacional a partir de las concepciones teóricas, permitiendo así la generación de conocimiento.

Con respecto a las técnicas manejadas en la investigación, se tienen: la observación documental, revisión en internet de revistas especializadas, material bibliográfico y la sistematización de la información. Así también se hizo uso de los instrumentos: block de notas, mapas conceptuales y Microsoft Office.

\section{Configuraciones Estructurales de las Formas Organizativas}

Los últimos años del siglo XIX y el siglo XX dejaron un cúmulo de aportes al desarrollo de las sociedades, se pasó de las actividades artesanales a la producción en masa y nació así el área industrial y con ello la incorporación de la tecnología a las ciencias, gracias a esto se acrecienta y consolida la relación que de manera conjunta llevan el hombre, la máquina y la producción, de igual forma se establecieron los estudios de los procesos y su gestión.

Hablar de la gerencia de las empresas, de su desarrollo y diseño organizacional, está vinculado con las ciencias económicas y sociales, en concordancia con lo que establece (Koontz, 2004) en la práctica, la administración es un arte y los conocimientos organizados en que se basa esta práctica son una ciencia, teniendo así un carácter complementario. Las diversas contribuciones de autores como Taylor, Weber, Drucker, entre otros, han dado como resultado los enfoques de la administración, abriendo paso a una evolución en la teoría administrativa. Este 
progreso del pensamiento administrativo se encuentra asociado a la transformación de las organizaciones y el mejoramiento de las condiciones de vida en la sociedad, así también es producto de la interacción entre los seres humanos, sus relaciones de poder y la productividad.

Las formas organizacionales generalmente tienden a surgir como respuesta a cambios en el desarrollo socioeconómico (Heydebrand, 1989); y según Minztberg (1991) citado por Padilla y Del Aguila (2003), estas se definen como configuraciones estructurales consistentes y altamente integradas. Por su parte Heydebrand (1989) las conceptualiza como un sistema de variables estructurales y propone seis dimensiones o variables para delinearlas o categorizarlas:

1) Tamaño de la fuerza de trabajo, referido al número de personas que integran la organización;

2) Objeto de trabajo, identificando si se diferencian por producir utilidades, proporcionar servicios o manipular símbolos a través del procesamiento de la información y de la toma de decisiones; 3) Medios de trabajo, que es la naturaleza de la herramientas, máquinas o instrumentos utilizados, y en general, el tipo de tecnología o el grado de complejidad tecnológica que emplean en la producción; 4) División del trabajo, es la composición ocupacional y de competencias de la fuerza de trabajo, así como la estructura administrativa de control tal y como ésta se manifiesta en la estructura departamental y divisional de las organizaciones; 5) Control de trabajo, se refiere a la naturaleza de la fuerza, la autoridad, la coordinación y el control administrativo en el nivel del proceso de trabajo o del punto de producción; y 6) Propiedad y control, son las relaciones sociales de producción en el nivel institucional y social.

Para entender las diversas formas organizacionales, interpretadas como representaciones que han surgido en función del diseño organizacional, las cuales pueden aludir a téminos distintos según la denominación de diferentes autores y estarse refiriendo a una misma configuración estructural, se describirán a continuación los puntos de vista expuestos por Chiavenato (2002) y Veciana (1999) con respecto a la relación entre el contexto histórico y el pensamiento administrativo; mientras que con Maldonado (2008), Padilla y Del Aguila (2003) se exponen sus propuestas de clasificación para las formas estructurales.

\section{Del Pensamiento Administrativo a la Organización}

Chiavenato (2002) presenta tres Eras en las que divide la evolución de la teoría administrativa y en las que muestra las diversas concepciones del hombre en las organizaciones, la Era Clásica (1900-1950), la Era Neoclásica (1950-1990) y la Era de la Información (1990Actualidad).

La Era Clásica, estuvo caracterizada por el establecimiento de teorías normativas y prescriptivas, gracias a las contribuciones de Taylor (1903), cuyos meritorios aportes sustentan los principios del área administrativa y la Ingeniería Industrial, con el planteamiento de la administración científica, los estudios de tiempos y movimientos, al igual que los métodos y procesos, centra su atención en el estudio de la administración desde la perspectiva de las tareas. Igualmente, se introducen los preceptos científicos, se incorporó la cooperación en el trabajo, maximizando la producción, seleccionando y distribuyendo a los trabajadores en concordancia a sus capacidades, aptitudes, atribuciones y responsabilidades, haciendo y adecuando el trabajo a los estudios humanos y de necesidades. 
Seguidamente Fayol, Gilbreth; Gantt, entre otros, postulan la Teoría Clásica, una teoría normativa y prescriptiva, cuyo objetivo se centra en la estructura de las organizaciones concentrando el objetivo de la misma en catorce principios y seis funciones administrativas. Posteriormente Weber (1947) incorpora la Teoría de la Burocracia, dándole el carácter normativo a la organización por medio del establecimiento de las reglamentaciones, la jerarquía y la autoridad pasan a direccionar los roles en el trabajo.

Paralelamente, para finalizar la Era Clásica, con la teoría de las Relaciones Humanas (1930), Hawthorme humaniza las organizaciones, entiende la relevancia de la comprensión del hombre como homo social y reivindica la importancia de gozar de unas buenas relaciones humanas en el ambiente laboral. De esta Era, se reconoce el salto de paradigma en visualizar el rol de hombre como ser humano y no como máquina, la conciencia de la necesidad de la producción, de la industrialización, pero a la vez de mejores métodos y prácticas que conlleven a una armonización integral entre el trabajo y el trabajador, merecen ser reconocidos como valiosos aportes a la ciencia administrativa.

Los cánones anteriormente descritos, citados de igual forma por Vargas y Nava (2009), marcan la forma de organización de las empresas, sientan las bases para la división de funciones y maneras de planificar el trabajo (Taylor, Fayol, Gilbreth, Gantt) y en consecuencia la mayoría de las empresas y organizaciones creadas en el siglo pasado, responde a este tipo de estructura formal, rígida, burocrática y jerárquica (Rincón y Romero, 2002). De esta forma el establecimiento de la cultura organizacional, las maneras de interactuar internamente y con el entorno, están direccionadas y se corresponden a la definición estructural como fueron concebidas estas empresas.

Según lo planteado por Chiavenato (2002) a mediados del siglo XX con la Era Neoclásica las teorías se convierten en enfoques del tipo explicativas y descriptivas, Drucker (1994) y Koontz (1980) determinan las funciones administrativas globalmente conocidas: planeación, organización, dirección y control, vigentes hasta el día de hoy. Por otra parte, surgen las teorías del comportamiento de Mc Gregor (1960) abriendo campo hacia la psicología organizacional, y consecutivamente, se inicia la tendencia que hace énfasis en la tecnología y el ambiente con la Teoría de Sistemas de Von Bertalanffy (1960). Dicha teoría entre sus postulados considera la visión de la totalidad, del todo a las partes y de las partes al todo, así como la visión causal de las partes integradas e interrelacionadas; también se adopta la visión funcional como principio teleológico.

Luego en 1970, Lawrence enuncia la Teoría Contingente, calificando a la organización como un ente contingente, con adaptabilidad y flexibilidad con el ambiente, abierta a la innovación y el mejoramiento continuo, esta organización reconoce la existencia de fuerzas externas sobre su desarrollo. Esta era representa una ruptura desde muchas perspectivas, los cambios sociales, políticos, tecnológicos, entre otros en forma conjunta también determinan nuevas formas de concebir las organizaciones, la teoría de sistemas se constituye como una teoría base para múltiples innovaciones posteriores; y abre paso a la Era de la Información.

A partir de los años 90, el dinamismo de los mercados y los avances tecnológicos dan propulsión a la Era de la Información, ésta se inicia colocando el énfasis en la tecnología, la 
transferencia del conocimiento, la productividad, la competitividad, el cliente y la globalización (Chiavenato, 2002) (Castells, 1998); concibiendo a la organización en un espacio en el cual no solo tiene valor lo tangible, en esta nueva era existe una apertura hacia la creación y adaptación de teorías, incluso de manera transdisciplinar, de forma tal que al observar la evolución de la teoría administrativa se representa un cambio de enfoque, un perfeccionamiento en la forma y perspectiva desde la cual abordar la organización, su funcionalidad, operación y sobrevivencia. Esta evolución se nutrió de los cambios de paradigmas y finalmente se estableció una interacción recíproca, global entre los cambiantes elementos: tareas, estructura, personas, tecnología y ambiente.

\section{La Visión de Organizar}

Conforme a lo expresado por Veciana (1999) en su libro "Función Directiva" la organización como tarea directiva involucra el establecimiento de un conjunto de normas configuradas racionalmente para establecer, regular y coordinar el proceso productivo. La tarea de organizar es concebida por este autor en cuatro etapas:

- Etapa del Taylorismo: se establece la organización científica del trabajo, se centra en un análisis de las clases de trabajo y su racionalización en estudios de tiempos y movimientos.

- Etapa Administrativa: a partir de Fayol la idea de organizar consiste en identificar, clasificar y distribuir de las unidades administrativas para el alcance de objetivos determinados.

- Etapa de Elthon Mayo: junto con Mc Gregor exponen que la organización debe centrarse en la interacción social, en las relaciones del grupo de trabajo (clima organizacional, relaciones informales, motivación) en donde la empresa tiene como fin satisfacer las necesidades de sus miembros.

- Etapa Actual: se destacan en esta etapa las teorías contingentes de la organización, según esta teoría no existe una única forma de organizar, el diseño de la estructura depende de su contexto o de una serie de factores propuestos por Chandler, Woordward, Lawrence, entre otros, en esta etapa se conjuga integralmente la eficiencia con los aspectos humanos

De esta forma se encuentran similitudes con Chiavenato en la distribución del pensamiento organizacional por etapas en función a los propuestos teóricos realizados en esos tiempos.

\section{Organizaciones Horizontales, en Red, Celular y Virtual}

Autores como Rivas (2002), definen las formas de las organizaciones como modelos organizacionales que se constituyen en reflejo de procesos racionales de configuraciones estructurales sensibles de ser gestionadas y que contribuyen en la definición del tipo de organización, su propósito y estrategias. Por lo tanto, presentan similitud con el concepto de forma organizacional que se ha venido utilizando en el presente artículo, Rivas (2002) presenta una tipología de formas denominadas por él como modelos organizacionales los cuales se basan en la evolución que tuvieron las teorías administrativas y los divide en tres espacios históricos: de 1900-1980 con la etapa de la estandarización; la idea era la búsqueda de la especialización, la producción en masa, los controles y el orden para lograr la eficiencia; 1981-1990 la etapa de la 
orientación al cliente y de 1991-a la actualidad, con la innovación. Esta evolución histórica difiere de la propuesta de Chiavenato (2002) en la progresión de la teoría administrativa en que concentra la Era Clásica junto con la Neoclásica, y toma la década de los 80 como parte de una segunda etapa, mientras que la Era de la Información es equivalente a la Innovación.

Rivas (2002), expresa que a partir de finales del siglo XX, aparecen los siguientes modelos o formas organizacionales: El modelo orgánico de Mintzberg (adhocracia y burocracia profesional); la organización horizontal, la organización tipo red, la organización celular y la organización virtual, sin embargo Wheelen (2007) incorpora en la organización en red a la organización virtual. Miller (1987) citado por Marín (2012) denominó al ambiente, la estrategia, la estructura y el liderazgo, como variables con poder influyentes y determinantes de la configuración organizacional, así como definió la existencia de variables estructurales y de estructuración.

Según Henry Mintzberg (1989), la organización puede ser dividida en cinco elementos que interactúan de acuerdo con los diferentes grados de incertidumbre que son la esencia de los procesos administrativos complejos. La organización dependerá de la conformación de sus grupos estratégicos, gerencia de línea, la tecno estructura, el staff y los grupos operativos. A partir de este modelo orgánico se pueden crear cinco estructuras: la estructura simple, (de tipo funcional); la burocracia mecánica; la burocracia profesional; la forma divisional y la adhocracia (Rivas, (2002)).

Se definen seguidamente las principales formas organizacionales:

- La Organización Horizontal: es otro tipo de diseño organizacional cuyo propósito es compactar las grandes estructuras tradicionalmente funcionales y verticales, la estructuración gira en torno a procesos y proyectos, desarrollados por equipos de alto desempeño (Rivas, 2002). Por su parte Chiavenato (2002) indica que este tipo de organización es de pocos niveles jerárquicos y presenta una configuración achatada, su trabajo es generalmente en equipos.

- La Organización en red, de acuerdo a Rivas (2002) está muy ligada al área tecnológica, ofrecen mayor adaptabilidad y flexibilidad al cambio rápido, está regida con los principios del pensamiento sistémico, donde la clave reside en la transferencia de conocimiento e información, generalmente estas estructuras son usadas en empresas con diferencias geográficas, el objetivo de trabajar en red es el establecimiento de interrelaciones colaborativas que buscan alcanzar metas corporativas específicas. Esta novedosa distribución rompe los esquemas de la jerarquía, la autoridad y la división del trabajo, se adecúa y adapta al dinamismo de los requerimientos del entorno.

Para describir el concepto de red organizacional en opinión de Moreno (2007) habría que ir más allá de lo morfológico para entrar a lo funcional. Puede entenderse como un grupo de nodos que se relacionan entre sí con ciertos fines explícitos, con una posible variedad de relaciones entre sus miembros, y cuya estructuración se basa en grados de descentralización (o agregación) de tareas junto con la toma democrática de decisiones (a menudo consensuada). En otras palabras, una red de carácter productivo; no puede ser un mero conjunto de contactos. Una 
red organizacional genera productos (que responden a objetivos) debe hacer algo, como un todo o a través de las múltiples subredes de geometría variable que pueden inscribirse en ella.

Además, Castells (1998) aduce que los procesos estructurantes de la economía, de la tecnología, de la comunicación, están cada vez más globalizados. Tal es el caso de los mercados financieros, de las redes productivas y comerciales de las principales empresas industriales, de los servicios estratégicos a las empresas (finanzas, publicidad, marketing), de los grandes medios de comunicación, de la ciencia y la tecnología. Este sistema global tiene estructura de red que, valiéndose de la flexibilidad proporcionada por las tecnologías de información, conecta todo lo que vale y desconecta todo aquello que no vale o se devalúa: personas, empresas, territorios, organizaciones. Así también, menciona como la nueva forma empresarial es la de redes de empresas, en las que se conectan departamentos de grandes empresas con una gran autonomía de decisión, con localizaciones de dichas empresas en distintos países, con redes de empresas colaboradores y alianza estratégicas, específicas para producto y función, tiempo y espacio, entre grandes empresas y sus redes respectivas (Harrison, 1996) citado por (Castells (1998)). También explica que esta organización en red tiene la ventaja de la flexibilidad, de la adaptación rápida a la demanda: cuando hay una demanda fuerte se organiza la red, cuando no la hay, se disuelve y se usan nuevos recursos.

Para complementar estas visiones, en este tipo de articulación Chiavenato (2002), indica que la organización en red separa sus funciones en compañías distintas conectadas a través de una organización central, en vez de juntar las funciones de producción, marketing, finanzas y RRHH en un mismo sitio, estos servicios son provistos por organizaciones separadas que trabajan por contrato y están conectadas electrónicamente a la oficina central.

Luego, producto de la evolución de la matriz y la red surge la Organización Celular, según Wheelen (2007) es una forma de organización compuesta por equipos autodirigidos, autoregulados, autoorganizados, responsables, colaborativos, con unidades de negocios autónomas, que interactúan de manera que conjugan la independencia con la interdependencia con otras células para producir a partir del conocimiento mayor poder, innovación y competitividad; se apoya en la teoría del caos y la teoría de sistemas.

- La Organización Virtual: Según Rivas (2002) es aquella que está conformada por grupos de organizaciones independientes relacionados por medio de redes electrónicas, temporales, no jerárquicas, carentes de estructura, cambiantes, con alto grado de virtualidad, vinculadas por la tecnología, los costos y la información, que buscan explotar una oportunidad de mercado.

Padilla y Del Águila (2003) destacan la organización virtual como evolución de la organización en red que responde a dos situaciones: la necesidad de que las estructuras organizativas sean más flexibles y la mayor implementación de estrategias de cooperación, que precisan de un soporte organizativo. Señalan de igual manera que para que la organización virtual funcione operativamente se precisa de Tecnologías de Información y Comunicación (TIC) que permitan la comunicación, colaboración y coordinación continúa de sus miembros. Esta organización virtual presenta como características las siguientes: dispersión espacial, uso 
intensivo de las TIC, concentración en las actividades fundamentales, alianzas estratégicas, virtualización de sus límites y subcontratación.

Por su parte Martínez y Cegarra (2014) describen para las empresas virtuales la conformación de un sistema de cadenas de valor relacionadas, entre proveedores, clientes, competidores, otras empresas y la misma organización, que conlleva una mayor eficiencia y sinergia para el sistema económico. Caracteriza a las empresas virtuales de la siguiente manera:

$\checkmark$ Se configura básicamente como una red de flujos de información, separando manifiestamente entre los flujos que están orientados a la acción, a los que están orientados a hacia la supervisión y al control de las operaciones.

$\checkmark$ Presentan una estructura de funcionamiento flexible, con capacidad de variabilizar relaciones y recursos, potenciando lo informal en torno a la formalidad del núcleo operativo.

$\checkmark$ Apuesta por la descentralización y por el mantenimiento de la eficacia operativa, sin menoscabo de la eficiencia organizativa.

$\checkmark$ Las relaciones organizativas y fronteras de la estructura se difuminan por efectos de la ruptura de la cadena de valor tradicional debido a los acuerdos de cooperación y alianzas.

La descripción de los modelos organizacionales anteriormente expuestos, en los cuales la estructura juega un rol principal, permite comprender la relevancia de distintos elementos como el tamaño, la especialización, la división del trabajo y departamentalización, la concentración de la autoridad en la toma de decisiones y la normalización, los cuales de manera sistémica contribuyen en la definición del tipo de organización.

\section{De Cara a la Post-Modernidad}

Velarde, Maldonado, et al. (2008) consideran como las características definitorias y diferenciadoras de la forma organizacional a las descritas como: estructura, objetivos, flexibilidad, uso de la tecnología, cultura, clima organizacional y participación de los trabajadores; las cuales son producto de tres enfoques organizacionales: premodernidad, modernidad y post-modernidad. Producto del análisis de las organizaciones desde la perspectiva de distintos autores, Velarde, Maldonado, et al. (2008) describen los criterios de postmodernidad que debe incluir la organización postmoderna, de la siguiente manera:

- El manejo de la operación de las organizaciones y la administración: empresa multiunitaria, administrada por directivos asalariados y profesionales, con unidades de operaciones independientes administrativa y financieramente, con empleados polivalentes, con estructuras flexibles.

- La importancia del elemento humano: empleo de por vida, evaluación y promoción, carreras no especializadas, mecanismos implícitos de control, proceso colectivo de toma de decisiones, responsabilidad colectiva, interés holista, alta tolerancia a la ambigüedad, alto compromiso institucional. Alta importancia a la calidad, flexibilidad, satisfacción de los clientes internos y externos, tecnología e información.

- La evaluación y la promoción: se considera primeramente los empleados de la organización que cumplen con el perfil. 
- Capacitación: alta especialización de la tarea basada en capacitación formal y alto contenido tecnológico.

- Baja formalización: como una de las características de la empresa adhocrática.

- La importancia en el servicio, la información y la calidad, satisfacción de los clientes internos y externos, tecnología de información.

A continuación en la Tabla 1 se resumen los momentos del pensamiento organizacional presentados previamente, en la cual se muestra la concepción del desarrollo de las empresas en función de las siguientes particularidades: contexto histórico, relación con el entorno, ciclos de la economía, objetivo y orientación de la organización, uso de los medios de producción. Cabe destacar que en función de esas distinciones nos encontramos actualmente en la Era de la información, la innovación y postmodernidad.

Tabla 1. Momentos del Pensamiento Organizacional.

\begin{tabular}{llll}
\hline \multicolumn{1}{c}{ Autor } & & \multicolumn{2}{c}{ Clasificación } \\
\hline Chiavenato (2002) & Era Clásica & Era Neoclásica & Era de la Información \\
Veciana (1999) & Etapa de Taylorismo & Etapa Administrativa & Etapa de Mayo Etapa Actual \\
& Estandarización & $\begin{array}{l}\text { Orientación al Cliente } \\
\text { Rivas (2002) }\end{array}$ & Innovación \\
Velarde, et al. (2003) & Pre Modernidad & Modernidad & Post Modernidad \\
\hline
\end{tabular}

Nota. Adaptación de Chiavenato (2002), Veciana (1999), Rivas (2002) Verlarde, et al. (2003)

\section{Nuevas Formas Organizativas}

De acuerdo a la definición dada por Padilla y Del Águila (2003) a las formas organizativas complejas nuevas se refieren a los nuevos planteamientos que se están haciendo con respecto a las configuraciones estructurales, estas proposiciones están centradas fundamentalmente, en explicar las nuevas formas organizativas que de un modo creativo adoptan las organizaciones para responder a los cambios del entorno actual. Entre sus aportes destacan el clasificar en simple, funcional, divisional, matricial, organización en red y organización virtual a estas configuraciones, destacando que a partir de las formas simple, funcional, divisional y matricial surgen un conjunto de derivaciones con sentido evolutivo, entre ellas destacan: la organización circular, en red, federal, basada en conocimiento, virtual, plana, horizontal, celular, entre otras, graficadas con carácter evolutivo a continuación. (Ver Figura 1) 


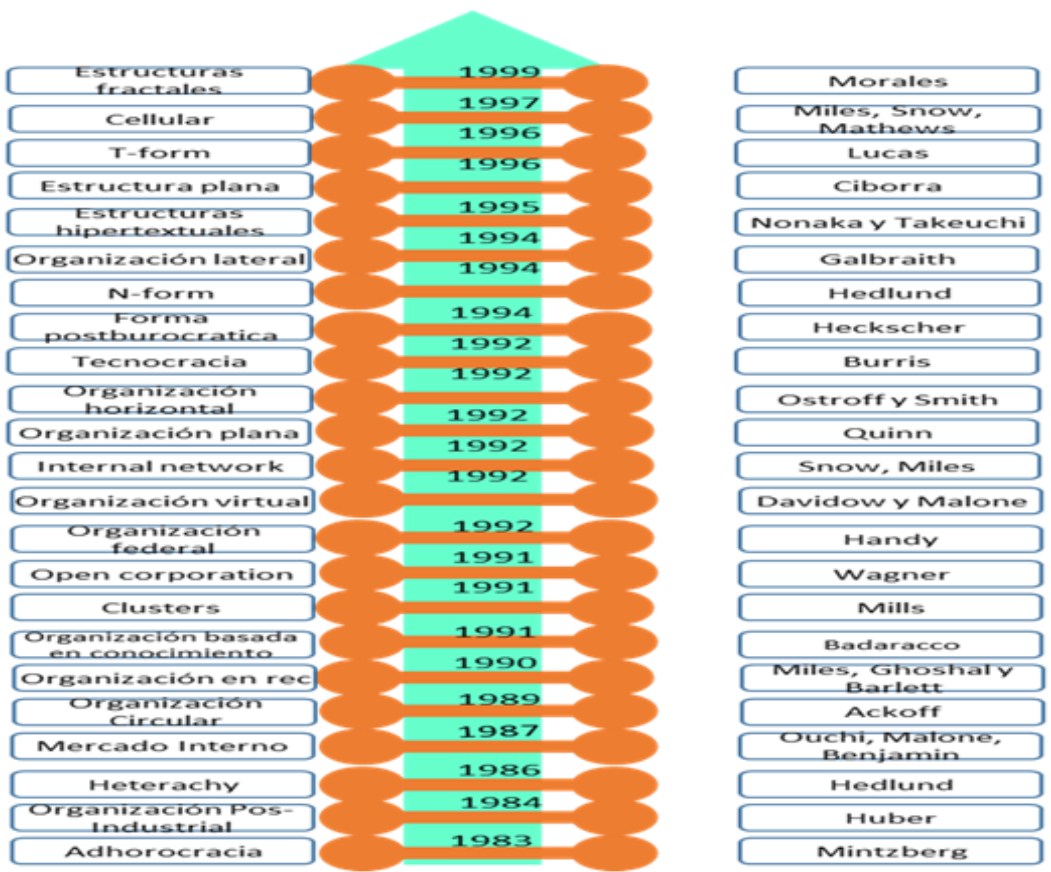

Figura 1. Sentido Evolutivo de las Nuevas Formas Organizativas.

Nota. Adaptación de Padilla, et al. (2003)

Padilla y Del Águila (2003) plantean que ningún modelo priva sobre otro, la escogencia de una forma organizacional obedece a los factores del contexto particular de cada empresa; en cuanto a la definición de las configuraciones no se ha producido un consenso general por parte de los autores en cuanto a la terminología, para una adecuada integración de las propuestas.

Una clasificación similar con respecto a las formas organizacionales es la presentada por Martínez y Cegarra (2014) en la que las clasifica como simples, complejas y complejas nuevas. Dentro de las simples ubica a las organizaciones con distribución funcional bien sea en línea o con modelo adhorocrático, regidas por el principio básico del diseño horizontal: la especialización. Seguidamente incluye como otro tipo de forma a las complejas subdivididas en líneo-funcional, multi-divisional, matricial y colegial, para finalmente plantear las complejas nuevas, con las formas en trébol, en red y federal.

Lo realmente resaltante de todas estas contribuciones sobre las formas organizacionales reside en la particularidad de los modelos de negocio, de los objetivos organizacionales propios, el nivel de especialización de su capital humano, el manejo de la autoridad y el contexto donde se desenvuelven, en consecuencia, una generalización o imposición de una forma específica no sería lo más conveniente, sin embargo, se observa la tendencia hacia el establecimiento de formas que permitan flexibilidad, adaptación, cambios, no sólo en estructura, funcionabilidad y operación sino en metas y objetivos que se plantean a nivel de las organizaciones.

\section{Propuesta Reflexiva}

Como consecuencia del análisis reflexivo de los planteamientos anteriores, se propone la integración con una visión de sistemas (Kast y Rosenzweig, 1998) e interrelacionada de diversos 
elementos como factores dinamizadores y acopladores de la forma organizacional; entre ellos se encuentran:

- Pensamiento estratégico: las tradiciones en las organizaciones se pueden convertir en una carga al momento de insertar una visión futurista alternativa, es necesario una minuciosa comprensión de la necesidad de concebir escenarios y acciones futuras, combinando el arte de la estrategia con la elección de futuribles. Pero el pensamiento estratégico no debe ser limitado a las unidades de mando o gerenciales, es requerido en todas las unidades organizacionales, para poder discriminar los indispensable, lo eventual y lo estratégico.

- Balance de recursos: la armoniosa combinación y articulación entre los recursos materiales, humanos y financieros, determina el éxito o el fracaso en el logro de los objetivos organizacionales. Los ciclos económicos y las limitaciones presupuestarias pueden condicionar las actividades productivas y la consecuente configuración estructural.

- Tecnología e Innovación: implementación de tecnologías y métodos de trabajo acordes a los nuevos tiempos, el diseño, adaptación e implementación de nuevas tecnologías además de contribuir con la productividad, proporcionan confiabilidad en los procesos. Incorporación de Internet como medio para interconectar sus procesos de trabajo y la actividad humana. (González, et al. 2009)

- Mejores Prácticas: la repetición de experiencias exitosas en procesos de trabajo realizadas con anterioridad en la empresa o en otras organizaciones, adecuándolas al contexto actual.

- Personal especializado: se requiere que el componente humano como esencia productiva posea habilidades intelectuales, conocimientos, técnicas y talentos para agregar valor al trabajo.(González, 2014)

- Participación Colectiva: tal como lo expresa Vargas y Nava (2009) la consecución de las metas empresariales demanda intervención y aportación de todos los integrantes de la organización con alto sentido de compromiso y pertenencia.

- Trabajo Colaborativo: los equipos de trabajo no solo deben ser efectivos sino ser autodirigidos (Becerra, 2010) y cooperar con todos los niveles organizativos para el bien común.

- Cultura Organizacional: los valores y conductas del personal no deben ser individualistas, el equilibrio del clima organizacional estará influenciado en gran medida por estos principios.

- Sistemas de Información y Comunicación: aunado al uso de TICs, se pretende integrar la divulgación oportuna de la información entre el personal con sistemas de información integrados e inteligentes (Parrales, 2017). 


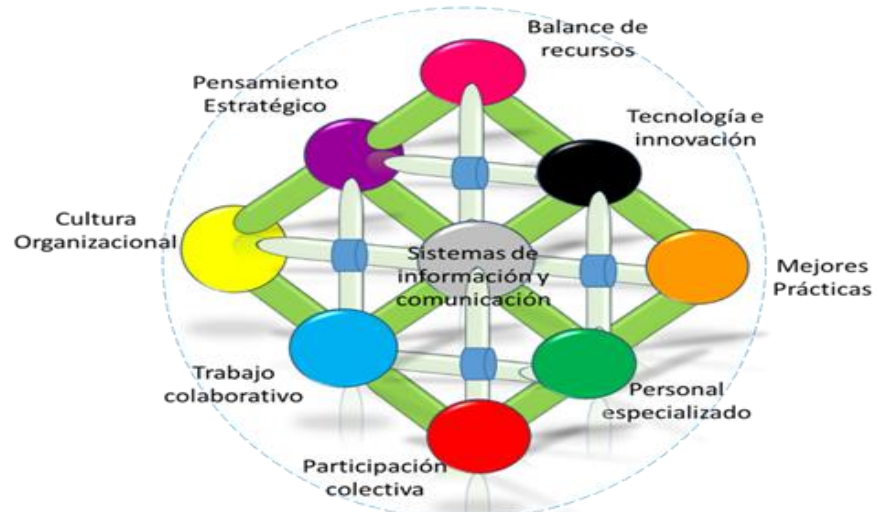

Figura 2. Propuesta de Elementos Internos Definitorios del Concepto Organizacional Nota. Elaboración Propia (2018).

Todos estos elementos se encuentran representados en la Figura 2 y constituyen los principios definitorios internos de la forma organizativa, la cual está inspirada en su estructura en las configuraciones en red y los diseños orgánicos, la componen a su vez unidades de negocios, departamentos, concebidos como sistemas y subsistemas abiertos e interrelacionados, ramificados con el personal de staff. Por otra parte, presenta semejanza en funcionamiento a la organización celular, como sistema abierto, (Morgan, 1990) define esta organización como un organismo vivo, el cual a su vez interactúa con el entorno de manera dinámica y en adaptación con sus colaboradores de acuerdo a los objetivos organizacionales propuestos. (Ver Figura 3)

Entre las características de la propuesta se incluyen: organización flexible, adaptable, con rápida capacidad de respuesta a los retos, eficiente, uso intensivo de las TICs, talento humano especializado, altamente formado, motivado y bien recompensado, con pensamiento estratégico, la autoridad es descentralizada, con sentido de responsabilidad compartida y con un proceso de toma de decisiones consensuado entre los miembros de la organización. En virtud de a la naturaleza de los negocios puede manejarse por objetivos o por proyectos, lo que requiere la gestión del personal con visión de colaboradores, los cuales pueden presentar una alta rotación.
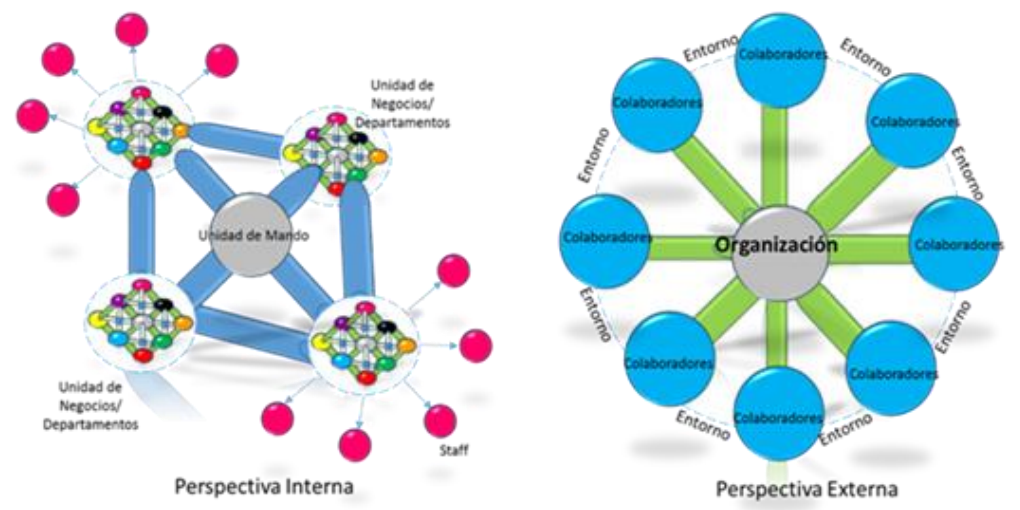

Figura 3. Perspectivas Internas y Externas con visión sistémica. Nota. Elaboración Propia (2018) 


\section{Conclusión}

La conformación de las empresas en diversas épocas de la historia contemporánea resultó exitosa debido a que se sustentaron de las sociedades a las que sirvieron, mantuvieron una interrelación estrecha con la puesta en práctica de las aportaciones teóricas realizadas en las ciencias administrativas, así también con las demandas que eran generadas; se dio paso así de una sociedad industrial con pensamiento mecanicista a una sociedad constituida por organizaciones tecnológicas, flexibles, adaptativas y complejas.

Las formas organizacionales horizontales y matriciales centran su perspectiva en la funcionalidad y departamentalización, poseen mayor grado de formalidad que las organizaciones en red y virtuales, cuyos principios reposan en el trabajo colaborativo, con toma de decisiones colectivas y personal con dominio técnico. Con el progreso de la tecnología y la globalización estas configuraciones estructurales seguirán avanzando y adaptándose a los requerimientos del mercado y la sociedad en general.

\section{Bibliografía}

Becerra, G. (2010). El liderazgo y su rol en las organizaciones transcomplejas. Visión Gerencial. Año 9, $\mathrm{N}^{\mathrm{o}}$ 1, Enero-Junio, 2010, pp. 30-42. Obtenido de: http://erevistas.saber.ula.ve/index.php/visiongerencial/article/view/799/798

Castells, M. (1998). Hacia el Estado Red? Globalización económica e instituciones políticas en la era de la información. Ponencia presentada en el Seminario sobre Sociedad y reforma del estado, Ministerio de Administración Federal e Reforma Do Estado, Sao Paulo. Obtenido de: https://uvirtual.udem.edu.co/file.php/2131/Documentos/Bibliografia/Basica/Tema3/Caste 11_Estado\%20Red.pdf

Chiavenato, I. (2002). Administración en los Nuevos Tiempos. Editorial Mc Grew Hill.

Heydebrand, W. (1989). New Organizational Forms. In Work and Occupations, vol. 16, no. 3, 1989, Sage Publications, pp. 323-357

Kast, F; Rosenzweig, J. (1998). Administración de las organizaciones: un enfoque de sistemas y de contingencias. México: McGraw-Hill.

Koontz, H. W. (2004). Administración una perspectiva global (7ma Edición). México: McGraw Hill.

González, I. (2014). Las Organizaciones Trans-Complejas: del Conocimiento a la Toma de

Decisiones. Revista Venezolana de Análisis de Coyuntura, vol. XX, núm. 1, enero-junio, 2014, pp. 235-246. Obtenido de: http://www.redalyc.org/pdf/364/36433515011.pdf

González, J; Pulgar, A; González, I. (2009). Uso de las TIC'S como Estrategia Gerencial para el Desarrollo de la Competitividad. Revista Científica Teorías, Enfoques y Aplicaciones en 
las Ciencias Sociales, Año 1, No. 2, 2009, págs. 85-98. Obtenido de. https://dialnet.unirioja.es/descarga/articulo/3990845.pdf

Marín, D. (2012). Estructura organizacional y sus parámetros de diseño: análisis descriptivo en pymes industriales de Bogotá. Estudios Gerenciales, Vol. 28, № 123, pp. 43-64. Abriljunio $2012 . \quad$ Obtenido de: https://www.icesi.edu.co/revistas/index.php/estudios_gerenciales/article/.../1207/1744

Martínez, A; Cegarra, J. (2014). Gestión por procesos de negocio. Organización Horizontal. Editorial del Economista. Ecobook. Obtenido de: www.ecobook.com > Libros

Moreno, A. (2007). Las organizaciones en red y sus nuevas identidades. Universidad Nacional de Educación a distancia. Facultad de Psicología. Tesis de Grado. Obtenido de: http://anamorenoromero.net/documentos/tesis_completa.pdf

Morgan, G. (1990). Imágenes de la Organización. RA-MA Editorial.

Padilla, A; Del Águila, A; (2003). La evolución de las formas organizativas. De la estructura simple a la organización en red y virtual. Investigaciones Europeas de Dirección y Economía de la Empresa. Vol. 9, $\mathrm{N}^{\mathrm{o}}$ 3, pp. 69-94. Obtenido de: https://dialnet.unirioja.es/descarga/articulo/793508.pdf

Parrales, J. (2017). Gerencia Estratégica y Tecnología de la Comunicación e Información- Tics. Revista Científica Dominio de las Ciencias. Vol. 3, Núm. Agosto 2017, pp. 218-236. Obtenido de: https://dialnet.unirioja.es/descarga/articulo/6102830.pdf

Rincón, D; Romero, M. (2002). Tendencias organizacionales de las empresas. Revista Venezolana de Gerencia, Vol. 7, N 19, Julio-Noviembre 2002, pp. 355-374. Obtenido de: http://www.redalyc.org/articulo.oa?id=29001902

Rivas, L. (2002). Nuevas Formas de Organización. Estudios Gerenciales. Universidad ICESI. Pp.13-45. Obtenido de: http://www.scielo.org.co/scielo.php?script=sci_arttext\&pid=S0123-59232002000100001

Vargas, J; Nava, S. (2009). Formas organizacionales, perfiles gerenciales y satisfacción laboral: un estudio descriptivo de PYMES en León, Guanajuato. Observatorio de la Economía Latinoamericana, $\quad \mathrm{N}^{\mathrm{O}} \quad 121, \quad 2009 . \quad$ Disponible en: http://www.eumed.net/cursecon/ecolat/mx/2009/vtna.htm

Veciana, J. (1999). Función directiva. Universidad Autónoma de Barcelona.

Velarde, M; Maldonado, A; Maldonado, M. (2008). El papel del elemento humano en la organización del sector turístico. Revista Gestión Turística, $\mathrm{N}^{\mathrm{o}}$ 10. Obtenido de: http://mingaonline.uach.cl/pdf/gestur/n10/art03.pdf 
Wheelen, T. Hunger, D. (2007). Administración Estratégica y Política de Negocios. Conceptos y casos. Décima edición, Pearson Educación. México. 